\title{
Relación entre la especie de Plasmodium y el grupo sanguíneo ABO: revisión sistemática
}

\author{
Relationship between Plasmodium species \\ and $A B O$ blood group: a systematic review
}

\author{
Santiago Rivera-Londoño $M D^{1}$, \\ Erika F. Garrido-Zea MSc²
}

Introducción: la malaria es una enfermedad tropical que es transmitida al ser humano por vectores (mosquitos) del género Anopheles, quienes transportan parásitos del género Plasmodium, encargados de producir la infección. En el mundo se presentan más de 219.000 .000 de casos y más de 660.000 muertes al año a causa de esta enfermedad. Múltiples estudios han demostrado que ciertas poblaciones son más susceptibles de presentar la infección y que dicha condición se relaciona con el grupo sanguíneo. Objetivo: describir la relación entre las especies de Plasmodium y el grupo sanguíneo ABO. Materiales y métodos: se realizó una revisión sistemática de artículos originales y revisión, publicados entre 1983 y 2015, en las bases de datos PubMed, Scielo y Elsevier. La búsqueda fue realizada en inglés y español y las palabras clave usadas para la búsqueda fueron "Whalgren» (estudioso de la enfermedad malárica), "malaria», "grupo sanguíneo $A B O »$, "Plasmodium falciparum», "RIFIN» (proteína expresada por Plasmodium falciparum) y «enfermedad malárica». Resultados: se encontraron un total de 50 artículos que hablan sobre generalidades de la enfermedad malárica y los grupos sanguíneos, la epidemiología de la enfermedad, el eritrocito como célula blanco para el parásito, la relación entre la malaria y el grupo sanguíneo y el fenómeno

\footnotetext{
${ }^{1}$ Estudiante de Medicina, Facultad de Ciencias de la Salud, Corporación Universitaria Remington. Medellín, Colombia. 2 Microbióloga y Bioanalista, MSc en Biología. Docente-Investigador, Facultad de Ciencias de la Salud, Corporación Universitaria Remington. Medellín, Colombia. Correspondencia: Calle 51 No 51-27. Correo electrónico: erika.garrido@uniremington.edu.co

Conflicto de intereses: los autores declaran que no tienen conflicto de intereses Medicina \& Laboratorio 2016; 22: 343-354

Módulo 7 (Microbiología), número 30. Editora Médica Colombiana S.A. $2016^{\circ}$

Recibido el 01 de junio de 2016; aceptado el 12 de agosto de 2016
} 
de rosetas, usados para la redacción de esta revisión. Conclusiones: las personas que presentan el grupo sanguíneo $O$ tienen menor riesgo de contraer la enfermedad malárica y su forma grave, causada principalmente por Plasmodium falciparum, en comparación con los que presentan fenotipo $A, B$ y $A B$.

Palabras clave: grupos sanguíneos, malaria, Plasmodium vivax, Plasmodium falciparum.

Introduction: Malaria is a tropical disease transmitted to human by Anopheles mosquito that transports parasites of Plasmodium gender, the causative agents of the infection. Worldwide more of 219.000 .000 cases and 660.000 deaths occur annually by this disease. Multiple studies have shown that certain populations are more sensitive for develop the infection, condition that have a relationship with the blood group. Objective: To describe the relationship between the Plasmodium species and the $A B O$ blood group. Materials and methods: A systematic review of original and review articles, published between 1983 and 2015, in PubMed, Scielo and Elsevier databases was performed. The search was conducted in English and Spanish and keywords used were "Whalgren" (expert on malaria disease), "malaria», "ABO blood type», "Plasmodium falciparum», «rifin» (protein expressed by Plasmodium falciparum), and «malarial disease». Results: It were found a total of 50 articles talking about generalities of malarial disease and blood groups, the epidemiology of the disease, the red blood cell as a target cell for the parasite, the relationship between malaria and blood group, and the erythrocyte rosetting phenomenon, used for writing this review. Conclusions: People with blood type $O$ have lower risk of malaria disease and its severe form, caused mainly by Plasmodium falciparum, compared with those with phenotype $A, B$ and $A B$.

Key words: Blood groups, malaria, Plasmodium vivax, Plasmodium falciparum.

Rivera-Londoño S, Garrido-Zea EF. Relación entre la especie de Plasmodium y el grupo sanguíneo ABO: revisión sistemática. Medicina \& Laboratorio 2016; 22: 343-354.

$\mathrm{L}$ a malaria es una enfermedad tropical transmitida al ser humano por vectores (mosquitos) del género Anopheles, cuya infección es producida por los parásitos del género Plasmodium, siendo los que infectan al humano Plasmodium vivax, Plasmodium falciparum, Plasmodium ovale, Plasmodium malarie y Plasmodium knowlessi [1].

Múltiples estudios demuestran que existen algunas poblaciones que tienen predisposición para adquirir infecciones, hecho que podría estar relacionado con el grupo sanguíneo. La malaria sirve como ejemplo para explicar esta condición, ya que al parecer se distribuye en la población dependiendo del grupo sanguíneo que tenga más frecuencia, como ha sido demostrado en regiones asiáticas y del África subsahariana [2-5].

El sistema sanguíneo $A B O$ es uno de los sistemas más utilizados en el mundo como marcador genético y al parecer tiene gran relevancia en lo que a malaria respecta. La distribución de los diferentes fenotipos de este grupo varía según la región geográfica y los grupos étnicos pertenecientes a dicha región. En regiones endémicas, 
por ejemplo, se ha reportado que el fenotipo $O$ es un factor protector para esta enfermedad mientras que el fenotipo $A$ y $B$ representan un factor de riesgo para contraerla y presentar la forma más severa $[3,6,7]$. Con base en lo anterior el objetivo de esta revisión fue describir la relación entre algunas especies de Plasmodium y el grupo sanguíneo $A B O$, utilizando diferentes medios bibliográficos.

\section{Materiales y métodos}

Se realizó una revisión sistemática de artículos originales y revisiones publicadas entre 1983 y 2015 en las bases de datos PubMed, Scielo y Elsevier, en idioma inglés y español, utilizando como criterio de búsqueda las palabras clave "Whalgren» (estudioso de la enfermedad malárica), "malaria», "grupo sanguíneo ABO», "Plasmodium falciparum», "RIFIN» (proteína expresada por Plasmodium falciparum) y «enfermedad malárica».

\section{Resultados}

Se encontraron un total de 50 artículos que según el periodo de publicación se distribuyen de la siguiente manera: 1983:1, 1990:1, 1994: 3, 1995: 1, 1998: 2, 2000: 1, 2004:2, 2005: 1, 2007: 2, 2008: 3, 2009: 4, 2010: 2, 2011: 5, 2012: 8, 2013: 3, 2014: 4, 2015: 4, 2016:3. Del total de artículos 10 hablan sobre generalidades tanto de la enfermedad malárica como de los grupos sanguíneos, 3 describen la epidemiología de la enfermedad, 8 hablan sobre el eritrocito como célula blanco para el parásito, 21 describen la relación entre la malaria y el grupo sanguíneo y 8 hablan sobre el fenómeno de rosetas. Estos artículos fueron utilizados para la redacción de este manuscrito (véase figura 1 y tabla $\mathbf{1}$ ).

\section{Generalidades sobre la malaria}

\section{Epidemiología de la malaria}

A nivel mundial se estima que se presentan más de 219.000 .000 de casos (43\% en África y $25 \%$ en el resto del mundo) y más de 600.000 muertes al año a causa de esta enfermedad $[2,30]$; sin embargo, según la Organización Mundial de la Salud, para el año 2014, el número de casos de malaria se redujo de 227.000 .000 en el 2000 a 198.000 .000 en 2013 [30].

En Latinoamérica el número de casos reportados ha descendido de 1.800 .000 en el 2000 a 526.000 en el 2009, siendo los dos países más endémicos del continente Brasil y Colombia, con un total hasta del $70 \%$ de todos los casos [30]. Lo anterior se debe a que la variedad climática en las diferentes regiones y subregiones de ambos países favorecen la reproducción del vector y por ende la aparición de la enfermedad, sobre todo en los lugares en los que es pobre el acceso al sistema de salud [31]. 


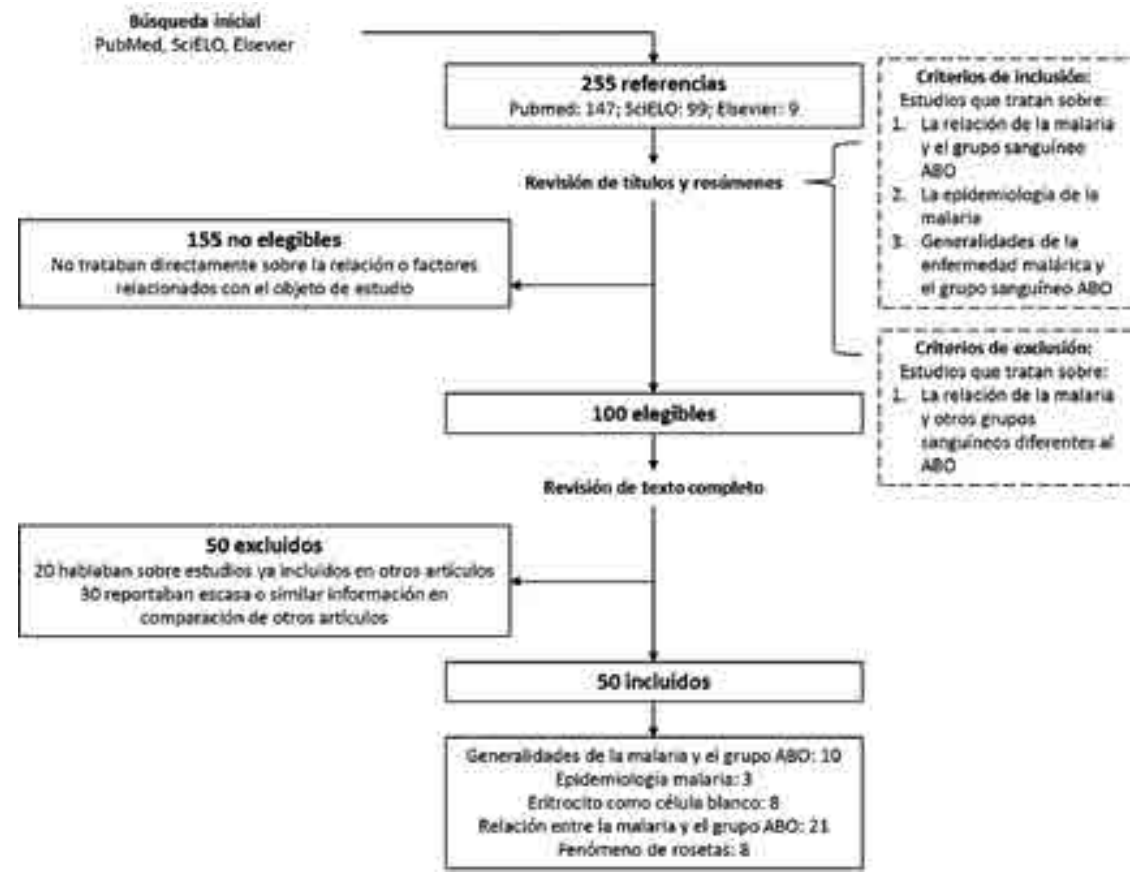

Figura 1. Estrategia de selección de artículos para la revisión sistemática.

\section{Tabla 1. Principales estudios incluidos en la revisión sistemática}

$\begin{array}{lll}\text { Autor (año) [ref.] } & \text { Población evaluada } & \text { Tipo de estudio } \\ \text { Anstee D.J. } & \text { No aplica } & \text { Revisión de } \\ \text { (2010) [8] } & & \text { tema }\end{array}$

Contenido/hallazgos principales

Anstee D.J. No aplica Revisión de

$\begin{array}{ll}\begin{array}{l}\text { Rowe y cols. Malí } \\ \text { (2009) [9] }\end{array} & \begin{array}{l}\text { Revisión de } \\ \text { tema }\end{array}\end{array}$

Rowe y cols. $\quad 67$ muestras de

(2007) [10] sangre de niños de Malí

Cserti-Gazde- 2.092 niños de wich y cols. (2012) [11]

Carlson y cols. Uganda (1994) [12] Uganda

Estudio de casos y controles

Estudio de casos y controles

Deepa y cols. (2011) [13]

Estudio de casos y controles

Estudio descriptivo-
100 pacientes en el St. John's Medical College Hospital, India
La predisposición a la malaria está dada por moléculas de expresión en la membrana del eritrocito tales como la glicoforina $\mathrm{C}$ que se presenta en las células del grupo sanguíneo $A$ y $\mathrm{B}$, por ello el $\mathrm{O}$ es un factor protector

Los individuos con grupos sanguíneos diferentes al O tiene un riesgo aumentado de tener malaria y su forma grave

De las muestras evaluadas se encontraron 124 niños con malaria complicada, de las cuales sólo el $21 \%$ pertenecían al grupo O. De este mismo grupo 14 niños fallecieron, entre los cuales sólo 2 tenían grupo sanguíneo O. Con base en lo anterior, se considera que el grupo $O$ es un factor protector contra la enfermedad El riesgo relativo de contraer malaria fue dos veces más alto para los grupos $A$ y $A B$ que para el grupo $\mathrm{O}$

Los grupos A y B tienen mayor capacidad de formar rosetas en contraste al grupo $\mathrm{O}$, pudiendo generar así la forma grave de la enfermedad comparativo
La proporción de pacientes que presentaron malaria severa y expresaban el antígeno 0 fue de $46 \%$, mientras que en los pacientes del grupo A la proporción de la infección severa fue de un $71 \%$ 


\begin{tabular}{|c|c|c|}
\hline $\begin{array}{l}\text { Rast y cols. } \\
(2004) \text { [14] }\end{array}$ & No aplica & $\begin{array}{l}\text { Revisión de } \\
\text { tema }\end{array}$ \\
\hline $\begin{array}{l}\text { Fry y cols. } \\
(2008) \text { [15] }\end{array}$ & $\begin{array}{l}4.000 \text { personas } \\
\text { de África sub- } \\
\text { sahariana }\end{array}$ & $\begin{array}{l}\text { Estudio de casos } \\
\text { y controles }\end{array}$ \\
\hline $\begin{array}{l}\text { Panda y cols. } \\
\text { (2011) [16] }\end{array}$ & $\begin{array}{l}453 \text { casos y } 174 \\
\text { controles sanos en } \\
\text { India }\end{array}$ & $\begin{array}{l}\text { Estudio de casos } \\
\text { y controles }\end{array}$ \\
\hline
\end{tabular}

\begin{tabular}{|c|c|c|}
\hline $\begin{array}{l}\text { Nasr y cols. } \\
(2012) \text { [17] }\end{array}$ & $\begin{array}{l}184 \text { individuos } \\
\text { de Kordofán del } \\
\text { Norte, Sudán }\end{array}$ & $\begin{array}{l}\text { Estudio } \\
\text { descriptivo- } \\
\text { comparativo }\end{array}$ \\
\hline $\begin{array}{l}\text { Akhigbe y } \\
\text { cols. (2011) } \\
\text { [18] }\end{array}$ & $\begin{array}{l}501 \text { estudiantes } \\
\text { de la Ladoke Anki- } \\
\text { tola University of } \\
\text { Technology (LAU- } \\
\text { TECH), Ogbomos- } \\
\text { ho, Nigeria }\end{array}$ & $\begin{array}{l}\text { Estudio de casos } \\
\text { y controles }\end{array}$ \\
\hline $\begin{array}{l}\text { Wolofsky y } \\
\text { cols. (2012) } \\
\text { [19] }\end{array}$ & $\begin{array}{l}\text { The Canadian In- } \\
\text { stitutes of Health } \\
\text { Research (CIHR), } \\
\text { Canadá }\end{array}$ & $\begin{array}{l}\text { Revisión siste- } \\
\text { mática }\end{array}$ \\
\hline $\begin{array}{l}\text { Singh }(2015) \\
y \text { cols. [20] }\end{array}$ & $\begin{array}{l}200 \text { muestras } \\
\text { analizadas en el } \\
\text { Mahatma Gandhi } \\
\text { Mission Medical } \\
\text { College, Navi } \\
\text { Mumbai, India }\end{array}$ & $\begin{array}{l}\text { Estudio } \\
\text { descriptivo- } \\
\text { comparativo }\end{array}$ \\
\hline $\begin{array}{l}\text { Barragan y } \\
\text { cols. (2000) } \\
{[21]}\end{array}$ & $\begin{array}{l}284 \text { pacientes de } \\
\text { Tailandia }\end{array}$ & $\begin{array}{l}\text { Estudio de casos } \\
\text { y controles }\end{array}$ \\
\hline $\begin{array}{l}\text { Fischer y } \\
\text { Boone (1998) }\end{array}$ & $\begin{array}{l}489 \text { pacientes de } \\
\text { Zimbawe }\end{array}$ & $\begin{array}{l}\text { Estudio de casos } \\
\text { y controles }\end{array}$ \\
\hline
\end{tabular}

controles sanos en y controles India
Relación de la proteína PfEMP1 con la enfermedad malárica

Las personas que expresaban los antígenos $\mathrm{A}$ (razón de disparidad $=1,33$ ) y $A B$ (razón de disparidad $=1,59$ ) tenían mucho más riesgo de contraer la forma grave de la infección, en comparación con los que expresaban el antígeno $\mathrm{O}$

La mayoría de los pacientes con malaria complicada y malaria severa pertenecían al grupo A, en contraposición al grupo O los cuales tenían un mayor número en el grupo de la enfermedad no complicada como en el de los controles sanos

El grupo O fue asociado con una disminución estadísticamente significativa del riesgo de contraer la forma grave de la enfermedad malárica

Se encontró que a pesar de que los individuos del grupo sanguíneo O eran más susceptibles de contraer la enfermedad malárica eran menos susceptibles de presentar la forma más severa de esta

Se observó un incremento en la fagocitosis por parte de los macrófagos en los glóbulos rojos con fenotipo $\mathrm{O}$ que estaban infectados y esto le fue atribuido al aumento de la deposición hemicrómica (fosfatidilserina) y la agregación de la banda 3

De 40 muestras 25 fueron positivas para Plasmodium vivax, 10 para Plasmodium falciparum y 5 para formas mixtas. Se concluyó que para los grupos $A, B$ y $A B$ hay, respectivamente, $2,5,2,5$ y 3,3 veces más riesgo de adquirir la infección por Plasmodium falciparum

Encontraron que tanto el antígeno A como el B pueden inducir una potenciación específica, pero no exclusiva de la formación de rosetas y que de estos dos, el grupo A es el que tiene más potencial de desarrollar la forma grave de la enfermedad

Los grupos A y B cuentan con unos trisacáridos A, GalNAca1-3(Fuca1-2) Gal1b1; y B, Gal1a1-3(Fuca1-2) Galb1, respectivamente, los cuales se han visto involucrados en el proceso de formación de rosetas y se encuentran ausentes en el grupo 0 , lo que explica la poca capacidad que tiene este grupo de producir dicho fenómeno

$\begin{array}{llll}\begin{array}{l}\text { Tadesse y Ta- } \\ \text { desse (2013) }\end{array} & \text { Etiopía } & \begin{array}{l}\text { Revisión de } \\ \text { tema }\end{array} & \begin{array}{l}\text { La presencia de moléculas de sulfato de con- } \\ \text { droitina A26 y CD3627 y el antígeno Duffy en } \\ \text { las células del grupo sanguíneo A promueven } \\ \text { una alta probabilidad de unión a las moléculas } \\ \text { de superficie para la formación de rosetas }\end{array} \\ \begin{array}{l}\text { Montoya y } \\ \text { cols. }(1994)\end{array} & \text { Colombia } & \begin{array}{l}\text { Revisión de } \\ \text { tema }\end{array} & \begin{array}{l}\text { El grupo sanguíneo B es más susceptible para } \\ \text { contraer la enfermedad malárica }\end{array}\end{array}$




\begin{tabular}{|c|c|c|c|}
\hline $\begin{array}{l}\text { Degarege y } \\
\text { cols. } \\
(2012) \\
{[25]}\end{array}$ & Etiopía & $\begin{array}{l}\text { Estudio trans- } \\
\text { versal }\end{array}$ & $\begin{array}{l}\text { Entre los participantes en el estudio, la } \\
\text { proporción de grupos sanguíneos O, A, B y } \\
\text { AB fue del } 40,1 \%, 30,1 \%, 29,0 \% \text { y } 14,3 \% \text {, } \\
\text { respectivamente, y los casos de malaria por } \\
\text { Plasmodium falciparum en los grupos sanguí- } \\
\text { neos correspondientes fueron } 14,8 \%, 14,0 \% \text {, } \\
13,4 \% \text { y } 15,7 \% \text {. Las probabilidades de malaria } \\
\text { no grave por Plasmodium falciparum no fueron } \\
\text { significativamente diferentes entre los indivi- } \\
\text { duos del grupo sanguíneo A frente a los O o } \\
\text { los B frente los O o de los AB frente a los O }\end{array}$ \\
\hline $\begin{array}{l}\text { Bamou y Sev- } \\
\text { idzem } \\
(2016) \text { [26] }\end{array}$ & $\begin{array}{l}\text { Universidad de Ds- } \\
\text { chang, Camerún }\end{array}$ & $\begin{array}{l}\text { Estudio de casos } \\
\text { y controles }\end{array}$ & $\begin{array}{l}\text { La prevalencia de malaria por Plasmodim falci- } \\
\text { parum fue del } 9,8 \% \text { y la mayoría de individuos } \\
\text { con grupo sanguíneo O estaban infectados } \\
\text { (15 casos, } 10,6 \% \text { ). No hubo relación entre } \\
\text { el grupo sanguíneo ABO y la prevalencia de } \\
\text { Plasmodium falciparum. Todos los individuos } \\
\text { que fueron positivos para la infección por } \\
\text { Plasmodium falciparum fueron asintomáticos }\end{array}$ \\
\hline $\begin{array}{l}\text { Amala y Nwi- } \\
\text { bani } \\
(2015) \text { [27] }\end{array}$ & $\begin{array}{l}\text { Agbonchia Health } \\
\text { Centre, Nigeria }\end{array}$ & $\begin{array}{l}\text { Estudio } \\
\text { descriptivo- } \\
\text { comparativo }\end{array}$ & $\begin{array}{l}\text { Se estudiaron } 250 \text { muestras de sangre de } \\
\text { niños, } 128(48,8 \%) \text { positivos para malaria. La } \\
\text { prevalencia de malaria por grupo sanguíneo } \\
\text { mostró que el grupo O tuvo la mayor prevalen- } \\
\text { cia }(24,0 \%) \text {, pero el menor número de casos } \\
\text { de malaria grave }(1,6 \%)\end{array}$ \\
\hline $\begin{array}{l}\text { Muntaka y } \\
\text { Opoku-Okrah } \\
(2013)[28]\end{array}$ & $\begin{array}{l}\text { Ghanaian Hospital, } \\
\text { Ghana }\end{array}$ & $\begin{array}{l}\text { Estudio } \\
\text { descriptivo- } \\
\text { comparativo }\end{array}$ & $\begin{array}{l}\text { Se examinaron } 437 \text { muestras, } 13 \% \text { positi- } \\
\text { vas para Plasmodium falciparum. El } 70,5 \% \\
\text { (308) eran mujeres y el } 29,5 \% \text { ( } 129) \text { eran } \\
\text { hombres. De los } 129 \text { donantes masculinos el } \\
19,4 \% \text { tenían malaria, mientras que sólo el } \\
10,4 \% \text { de las mujeres la tenía. Las tasas de } \\
\text { infección para los grupos sanguíneos A, B, O } \\
\text { y AB fueron } 14,3 \%, 11,1 \%, 13,9 \% \text { y } 0,0 \% \text {, } \\
\text { respectivamente }\end{array}$ \\
\hline $\begin{array}{l}\text { Nkuo-Akenji } \\
\text { y cols. (2004) } \\
\text { [29] }\end{array}$ & Camerún & $\begin{array}{l}\text { Estudio } \\
\text { descriptivo- } \\
\text { comparativo }\end{array}$ & $\begin{array}{l}\text { Se estudiaron } 121 \text { pacientes febriles de } 1-60 \\
\text { años de edad. De acuerdo al grupo sanguíneo } \\
\text { se encontró la mayor prevalencia de malaria } \\
(74,5 \%) \text { en los individuos del grupo O y la } \\
\text { menor }(58,6 \%) \text { en los individuos del grupo B }\end{array}$ \\
\hline
\end{tabular}

\section{Aspectos generales de la malaria y el sistema $A B O$}

\section{Sistema ABO}

En 1900, Landsteiner y colaboradores descubrieron que los eritrocitos se pueden clasificar en A, B y O, de acuerdo a la presencia o ausencia de antígenos [32,33], los cuales se encuentran adheridos a la membrana de los glóbulos rojos y cuya especificidad está dada por la presencia de un monosacárido terminal. De esta manera, el monosacárido $\mathrm{N}$-acetil-galactosamina determina la especificidad antigénica $A$ mientras que el monosacárido galactosa determina la especificidad del $B$; entretanto, el grupo $O$ se caracteriza porque no expresa el azúcar terminal de $A$ ni de $\mathrm{B}$. Los antígenos $\mathrm{ABO}$ están presentes en todos los tejidos excepto el sistema nervioso central [32]. 


\section{El eritrocito como célula blanco para Plasmodium spp.}

Plasmodium spp. tiene un ciclo de vida complejo que se desarrolla en diferentes fases, tanto en el mosquito Anopheles (vector) como en el humano (hospedero). En este último, las formas llamadas esporozoitos, presentes en las glándulas salivales del mosquito, son inyectadas al torrente sanguíneo y viajan hasta llegar al hígado y ubicarse en los hepatocitos. Después de un periodo de 10 a 12 días el hepatocito se revienta y libera las formas llamadas merozoítos, las cuales invaden los glóbulos rojos. Allí, el parásito lleva a cabo el ciclo intraeritrocítico, en el cual, además de generarse los gametocitos, encargados de mantener la transmisión de la enfermedad hacia el mosquito, se liberan los merozoítos a la sangre, los cuales infectan nuevos eritrocitos sanos $[34,35]$.

En estudios in vitro se han encontrado algunas moléculas que son capaces de mediar la adherencia del parásito al glóbulo rojo, actuando como receptores. Entre estos, los más importantes en el endotelio son la glucoproteína CD36 y la molécula de adhesión intercelular-1 (ICAM-1; del inglés, Intercellular Adhesion Molecule-1), y en la placenta el sulfato A de condroitina [11]. Otros receptores incluyen la molécula de adhesión vascular-1 (VCAM-1; del inglés, Vascular Cell Adhesion Molecule-1) y la E-selectina en el endotelio, las proteínas PECAM-1/ CD31 en las plaquetas y el endotelio, el sistema de antígenos $A B O$, el sulfato de heparina (o heparán sulfato) y el receptor del complemento tipo 1 (CR1) en los eritrocitos no parasitados [36]. Otro estudio realizado en la República Democrática del Congo, África, demostró que en la superficie del eritrocito se expresa el receptor glicoforina $\mathrm{B}$ que se une a la proteína EBL-1 del Plasmoduim falciparum [37].

Por su parte, los ligandos PfRH (del inglés, Reticulocyte Binding Protein Homolog) están localizados en la proteína roptrias de los merozoítos y se dividen en PfRH1, PfRH2a, PfRH2b, PfRH4 y PfRH5. Específicamente, el ligando PfRH4, a diferencia de los antes mencionados, es esencial para mediar la entrada del Plasmodium falciparum a los glóbulos rojos humanos mediante la unión al receptor del complemento tipo 1 . Un estudio demostró que se podían desarrollar anticuerpos contra esta proteína, lo cual permitiría la inhibición de la invasión por parte de esta especie de Plasmodium [38,39].

Las personas que son parasitadas por Plasmodium spp. tienen una mayor expresión de moléculas de adhesión tales como ICAM-1, E-selectina y VCAM-1 tanto en el endotelio como en otros tejidos y el plasma, permitiendo así que el parásito siga invadiendo otros glóbulos rojos y perpetúe su ciclo $[23,36]$. Cuando Plasmodium falciparum parasita los glóbulos rojos codifica un ligando llamado PfEMP1 (del inglés, Plasmodium falciparum Erythrocyte Membrane Protein-1) que se encarga de unir el glóbulo rojo infectado con uno no infectado facilitando un mayor grado de invasión [40]. 


\section{Relación de la malaria y el grupo sanguíneo ABO}

El grupo sanguíneo $A B O$ y su relación con la malaria ha sido estudiado desde hace algunas décadas y por ello existe numerosa evidencia sobre esta relación [8]. Dichos estudios han demostrado que el grupo A puede representar un riesgo para la enfermedad mientras que el grupo O confiere protección [9-14].

En Ogbomosho, Nigeria se realizó un estudio en 501 estudiantes de la Ladoke Ankitola University of Technology (LAUTECH), en el que se encontró que, a pesar de que algunos individuos que presentaban el grupo sanguíneo $O$ eran susceptibles de contraer la enfermedad malárica tenían menos susceptibilidad a presentar la forma más severa de esta [18].

Un estudio realizado por Fry y colaboradores (2008) [15] en el África subsahariana, que tomó al menos 4.000 individuos con malaria grave, describió que las personas que expresaban los antígenos $A$ y $A B$ tenían mucho más riesgo de contraer la forma grave de la infección (razón de disparidad=1,33 y 1,59, respectivamente), en comparación con los que expresaban el antígeno $\mathrm{O}$. No obstante, no se sabe exactamente si esta protección depende exclusivamente de dicho antígeno, ya que en otras regiones se han observado individuos con grupo sanguíneo $O$ que padecen malaria. Lo anterior se podría explicar por la presencia de otros componentes que representan un factor de riesgo para contraer la infección, por ejemplo, la variación en los niveles de transmisión e inmunidad de la población de estudio $[11,16,17,24,36]$.

En el St. John's Medical College Hospital en Bangalore, India, se realizó un estudio en 2007 que reunió 100 pacientes con diagnóstico de malaria, de los cuales 22 eran A positivo, 42 B positivo, 35 O positivo y uno AB positivo. La mayoría de los casos fueron por Plasmodium falciparum (61\%) en contraste con Plasmodium vivax (37\%). La proporción de pacientes que presentaron malaria severa y expresaban el antígeno O fue del $46 \%$, en comparación con los pacientes del grupo A, en los cuales la proporción fue del $71 \%$ [13].

En un estudio realizado por The Canadian Institutes of Health Research (CIHR) en Canadá se demostró, tanto in vivo como in vitro, que el desarrollo de los merozoítos era igual para los fenotipos $A B O$, pero se observó un incremento en la fagocitosis por parte de los macrófagos de los glóbulos rojos con fenotipo $O$ infectados, que fue atribuido al aumento de la deposición hemicrómica (fosfatidilserina) y la agregación de banda 3, pues, a pesar de que en los glóbulos rojos no infectados estas características eran iguales para los fenotipos $\mathrm{A}, \mathrm{B}$ y $\mathrm{O}$, en los infectados eran mayores en el grupo O [19].

Otro estudio realizado en el Departamento de Microbiología del Mahatma Gandhi Mission Medical College and Hospital en Navi Mumbai, India, incluyó 200 muestras de pacientes con sospecha de malaria, de las cuales 40 fueron positivas para la enfermedad y 160 negativas. De las 40 muestras positivas 25 fueron para Plasmodium 
vivax, 10 para Plasmodium falciparum y cinco para formas mixtas. Como conclusión se obtuvo que para los grupos A, B y $A B$ hay, respectivamente, 2,5, 2,5 y 3,3 veces más riesgo de adquirir la infección por Plasmodium falciparum [20].

\section{Grupo ABO en relación con la formación de rosetas}

La formación de rosetas es un factor de virulencia de Plasmodium falciparum que consiste en la capacidad que el parásito le confiere a los glóbulos rojos infectados de unirse con los glóbulos rojos no infectados [41,42]. Para que este proceso se lleve a cabo se necesita de un receptor que proviene de un subgrupo de adhesinas conocido como PfEMP1 $[15,43,44]$. Esta proteína es codificada por el genoma del parásito y expresada en la superficie de los glóbulos rojos infectados, donde actúa como un sitio de unión a ciertos receptores blanco que se encuentran en la superficie de los glóbulos rojos, las plaquetas y las células vasculares endoteliales $[40,45]$.

Estructuralmente la pfEMP1 tiene dos dominios principales: el DBL (del inglés, Duffy-binding-like domains) que se une de manera primaria a los oligosacáridos de los grupos sanguíneos A y B, y el CIDR1 (del inglés, Cysteine-Rich Interdomain Regions-1) que se une a las plaquetas y el endotelio, permitiendo así el proceso de formación de rosetas $[40,45]$. A pesar de que Plasmodium falciparum es el único entre las especies causantes de malaria que puede inducir la producción de la proteína PfEMP1 en los eritrocitos infectados, en un estudio realizado en 2014 se demostró que Plasmodium vivax también puede inducir el proceso de formación de rosetas mediante un receptor llamado glicoforina C (CD236R) [46]; aunque en dicho estudio se concluyó que aún hace falta hallar ligandos que se relacionen con esta molécula y otros receptores que expliquen la inducción de dicho fenómeno por esta especie de Plasmodium $[12,46,47]$.

Barragan y colaboradores (2000) [21] confirmaron que tanto el antígeno A como el $B$ pueden inducir una potenciación específica, pero no exclusiva, de la formación de rosetas y que, de estos dos, el grupo $A$ es el que tiene más potencial de desarrollar la forma grave de la enfermedad. Por su parte, el antígeno $O$ tiene un potencial muy bajo para desarrollar este fenómeno $[13,48,49]$. Los grupos $A$ y B cuentan con unos trisacáridos A, GalNAca1-3(Fuca1-2) Gal1b1 y B, Gal1a13(Fuca1-2) Galb1, respectivamente, quienes se han visto involucrados en el proceso de formación de rosetas y su ausencia en el grupo O explica la poca capacidad que tienen estos glóbulos rojos de producirlo $[16,22,50]$.

Así mismo, la presencia de varias moléculas glicosiladas de adhesión intracelular, moléculas de sulfato de condroitina A26 y CD3627 y el antígeno Duffy en células del grupo sanguíneo A promueven una alta probabilidad de unión a las moléculas de superficie para la formación de rosetas por parte de Plasmodium falciparum, conduciendo así al desarrollo de la malaria grave [23]. 


\section{Discusión}

La relación entre el grupo sanguíneo $A B O$ y la malaria ha venido siendo reportada en múltiples estudios que datan desde el siglo pasado hasta el presente año. Dicha relación nos ayuda a comprender más allá sobre la forma en la que se presenta la infección y su forma más grave en el ser humano. En múltiples estudios se ha demostrado que el grupo A y B representan factores de riesgo para contraer la enfermedad, ya que en su membrana cuentan con unos trisacáridos que sirven de receptores para el parásito, en contraposición al grupo $\mathrm{O}$ que al no presentar estos trisacáridos en su membrana adquiere protección frente a la malaria [16].

En otros estudios se habla de otros receptores tales como la glucoproteína CD36, la ICAM1, el sulfato A de condroitina, la VCAM-1 y la E-selectina, entre otras, cuya función es mediar la adhesión de los ligandos del parásito al glóbulo rojo [36]. Un ejemplo es el receptor del complemento tipo 1 (CR1) al cual se une el ligando PfRH4 de Plasmodium falciparum, permitiendo así la invasión del eritrocito [38]. En el caso de Plasmodium vivax la unión al eritrocito se da a través de un receptor llamado glicoforina C (CD236R) [46].

Aunque los individuos de los grupos sanguíneos A y B se han visto más comprometidos por la enfermedad malárica se ha observado que hay poblaciones con personas que expresan el grupo $O$ en las que no sólo se presenta la enfermedad, sino su forma más grave, pero en menor proporción si se compara con aquellos del grupo A o B. Lo anterior puede ser explicado por diferentes factores como la variación en los niveles de inmunidad de dichas poblaciones $[11,36]$.

El proceso de formación de rosetas se ha descrito en los casos de infección por el Plasmodium falciparum, pero en algunos estudios se ha demostrado su desarrollo por Plasmodium vivax. Cuando el glóbulo rojo es parasitado por Plasmodium falciparum se expresa una proteína de unión llamada PfEMP1, la cual con su dominio DBL se une a los oligosacáridos expresados en el grupo $A$ y $B$, y con su dominio CIDR1 se une a las plaquetas y el endotelio, permitiendo así el secuestro del glóbulo rojo a nivel vascular con la consiguiente hipoxia y daño tisular; entendiéndose esto como la forma grave de la enfermedad. Lo anterior toma relevancia ya que esta adhesina no se va a unir al eritrocito del grupo $O$ puesto que no tiene los oligosacáridos que expresan los grupos $A$ y $B$, haciendo que este sea un factor protector contra la forma grave de la malaria $[40,45]$.

La relación entre la malaria y el grupo sanguíneo $A B O$ es de gran relevancia no sólo como una forma de entender la fisiopatología de la enfermedad, sino también de establecer claramente cuáles individuos tienen mayor riesgo de contraer la infección y de padecer su forma más grave; dicho conocimiento permitiría iniciar esquemas terapéuticos óptimos encaminados a limitar en los pacientes el desarrollo de la malaria grave, lo que se vería reflejado en un descenso en las cifras de morbimortalidad para la malaria. 


\section{Conclusiones}

Múltiples estudios han demostrado que existe una relación entre la malaria y el grupo sanguíneo $A B O$, que se explica por varios factores entre los cuales se incluye la expresión de ciertos oligosacáridos en la membrana de los eritrocitos de los grupos sanguíneos A y $\mathrm{B}$, que actúan como receptores para los ligandos de los agentes causales de la malaria, Plasmodium falciparum y Plasmodium vivax. Con lo anterior, se entiende que los eritrocitos del grupo sanguíneo $\mathrm{O}$ al no presentar estos antígenos adquieren protección frente a la infección y la forma más grave de esta enfermedad. Cabe resaltar que a pesar de que muchos estudios hablan sobre Plasmodium falciparum pocos lo hacen sobre Plasmodium vivax, por lo que se requieren más estudios en esta especie para así establecer una relación más concreta con el grupo sanguíneo ABO.

\section{Bibliografía}

1. Organización Mundial de la Salud. Paludismo. Nota descriptiva No. 94. 2016. Disponible: http:// www.who.int/mediacentre/factsheets/fs094/es/. Consultado: jun 2016.

2. Organización Panamericana de la Salud. Informe de la Situación del Paludismo en las Américas. Washington D.C., Estados Unidos: OPS; 2008.

3. Almaguer LE, Betancourt P. Genética poblacional para el sistema sanguíneo $A B O$ en una población con malaria endémica. CCM 2014; 18: 1-10.

4. González-Ordóñez ÁJ. Grupos sanguíneos y enfermedad. Med Clin (Barc) 2005; 125: 382-388.

5. Cserti CM, Dzik WH. The ABO blood group system and Plasmodium falciparum malaria. Blood 2007; 110: 2250-2258

6. Lalueza-Fox C, Gigli E, de la Rasilla M, Fortea J, Rosas A, Bertranpetit J, et al. Genetic characterization of the $A B O$ blood group in Neandertals. BMC Evol Biol 2008; 8: 1-5.

7. Tekeste Z, Petros B. The ABO blood group and Plasmodium falciparum malaria in Awash, Metehara and Ziway areas, Ethiopia. Malar J 2010; 9: 280.

8. Anstee DJ. The relationship between blood groups and disease. Blood 2010; 115: 4635-4643.

9. Rowe JA, Opi DH, Williams TN. Blood groups and malaria: fresh insights into pathogenesis and identification of targets for intervention. Curr Opin Hematol 2009; 16: 480-487.

10. Rowe JA, Handel IG, Thera MA, Deans AM, Lyke KE, Koné A, et al. Blood group O protects against severe Plasmodium falciparum malaria through the mechanism of reduced rosetting. Proc Natl Acad Sci USA 2007; 104: 17471-17476.

11. Cserti-Gazdewich CM, Dhabangi A, Musoke C, Ssewanyana I, Ddungu H, Nakiboneka-Ssenabulya $\mathbf{D}$, et al. Cytoadherence in paediatric ma- laria: ABO blood group, CD36, and ICAM1 expression and severe Plasmodium falciparum infection. $\mathrm{Br}$ J Haematol 2012; 159: 223-236.

12. Carlson J, Nash GB, Gabutti V, al-Yaman F, Wahlgren $\mathbf{M}$. Natural protection against severe Plasmodium falciparum malaria due to impaired rosette formation. Blood 1994; 84: 3909-3914.

13. Deepa, Alwar VA, Rameshkumar K, Ross C. ABO blood groups and malaria related clinical outcome. J Vector Borne Dis 2011; 48: 7-11.

14. Rast N, Wahlgren $\mathbf{M}$, Chen $\mathbf{Q}$. Molecular aspects of malaria pathogenesis. FEMS Immunol Med Microbiol 2004; 41: 9-26.

15. Fry AE, Griffiths MJ, Auburn S, Diakite M, Forton JT, Green A, et al. Common variation in the $A B O$ glycosyltransferase is associated with susceptibility to severe Plasmodium falciparum malaria. Hum Mol Genet 2008; 17: 567-576.

16. Panda AK, Panda SK, Sahu AN, Tripathy R, Ravindran B, Das BK. Association of ABO blood group with severe falciparum malaria in adults: case control study and meta-analysis. Malar J 2011; 10: 309.

17. Nasr A, Eltoum M, Yassin A, ElGhazali G. Blood group $O$ protects against complicated Plasmodium falciparum malaria by the mechanism of inducing high levels of anti-malarial IgG antibodies. Saudi J Health Sci 2012; 1: 16-22.

18. Akhigbe RE, Ige SF, Adegunlola GJ, Adewumi MO, Azeez MO. Malaria, Haemoglobin Genotypes and $\mathrm{ABO}$ Blood groups in Ogbomoso, Nigeria. Int J Trop Med 2011; 6: 73-76.

19. Wolofsky KT, Ayi K, Branch DR, Hult AK, Olsson ML, Liles WC, et al. ABO blood groups influence macrophage-mediated phagocytosis of Plasmodium falciparum-infected erythrocytes. PLoS Pathog 2012; 8: e1002942.

20. Singh G, Urhekar AD, Singh R. A study on correlation of malaria infection with $A, B, O$, RH blood 
group system. J Parasitol Vector Biol 2015; 7: 6773.

21. Barragan A, Kremsner PG, Wahlgren M, Carlson $\mathbf{J}$. Blood group $A$ antigen is a coreceptor in Plasmodium falciparum rosetting. Infect Immun 2000; 68: 2971-2975.

22. Fischer PR, Boone $\mathbf{P}$. Short report: severe malaria associated with blood group. Am J Trop Med Hyg 1998; 58: 122-123.

23. Tadesse $\mathbf{H}$, Tadesse $\mathbf{K}$. Assessing the association of severe malaria infection and $A B O$ blood groups in northwestern Ethiopia. J Vector Borne Dis 2013; 50: 292-296.

24. Montoya F, Restrepo M, Montoya AE, Rojas W. Blood groups and malaria. Rev Inst Med Trop Sao Paulo 1994; 36: 33-38.

25. Degarege A, Medhin G, Animut A, Legess M Erko B. Association of $A B O$ blood group and $P$. falciparum malaria related outcomes: a cross-sectional study in Ethiopia. Acta Trop 2012; 123: 164 169.

26. Bamou R, Sevidzem SL. ABO/Rhesus blood group systems and malaria prevalence among students of the University of Dschang, Cameroon. MWJ 2016; 7: 1-4.

27. Amala SE, Nwibani CP. Malaria in children, its association with $\mathrm{ABO}$ blood group and haemoglobin genotype. IJDR 2015; 5: 5958-5962.

28. Muntaka S, Opoku-Okrah C. The Prevalence of Malaria Parasitaemia and Predisposition of $A B O$ Blood Groups to Plasmodium falciparum Malaria among Blood Donors at a Ghanaian Hospital. AU JT 2013; 16: 255-260.

29. Nkuo-Akenji TK, Wepngong $P$, Akoachere JF. Effects of ABO/Rh blood groups, G-6-P-D enzyme activity and haemoglobin genotypes on malaria parasitaemia and parasite density. Afr J Health Sci 2004; 11: 93-97.

30. World Health Organization. World Malaria Report. Ginebra, Suiza: WHO Press. 2014.

31. Padilla-Rodríguez JC, Álvarez-Uribe G, Montoya-Araújo R, Chaparro-Narváez $P$, Herrera-Valencia $\mathbf{S}$. Epidemiology and control of malaria in Colombia. Mem Inst Oswaldo Cruz 2011; 106: $114-122$.

32. Grispan DS. Grupos sanguíneos $A B O$ y Rh. Rev Medica Hondur 1983; 51: 103-114.

33. Storry JR, Olsson ML. The ABO blood group system revisited: a review and update. Immunohematology 2009; 25: 48-59.

34. Vásquez AM, Tobón Alberto. Mecanismos de patogenia en la malaria por Plasmodium falciparum. Biomédica 2012; 32: 106-120.

35. Centers of Disease Control and Prevention (CDC). Malaria: Biology. 2016. Disponible: www. cdc.gov/malaria/about/biology/. Consultado: jun 2016 .

36. Rowe JA, Claessens A, Corrigan RA, Arman M. Adhesion of Plasmodium falciparum-infected erythrocytes to human cells: Molecular mechanisms and therapeutic implications. Expert Rev Mol Med 2009; 11: e16.
37. Mayer DCG, Cofie J, Jiang L, Hartl DL, Tracy E, Kabat J, et al. Glycophorin B is the erythrocyte receptor of Plasmodium falciparum erythrocyte-binding ligand, EBL-1. Proc Natl Acad Sci USA 2009; 106: 5348-5352.

38. Reiling L, Richards JS, Fowkes FJ, Wilson DW, Chokejindachai W, Barry AE, et al. The Plasmodium falciparum Erythrocyte Invasion $\mathrm{Li}$ gand Pfrh4 as a Target of Functional and Protective Human Antibodies against Malaria. PLoS One 2012; 7: e45253.

39. Lim NT, Harder MJ, Kennedy AT, Lin CS, Weir C, Cowman AF, et al. Characterization of inhibitors and monoclonal antibodies that modulate the interaction between Plasmodium falciparum adhesin PfRh4 with its erythrocyte receptor Complement Receptor 1 J Biol Chem 2015; 290: 25307-25321.

40. Kuadzi JT, Ankra-Badu G, Addae MM. Plasmodium falciparum malaria in children at a tertiary teaching hospital: $A B O$ blood group is a risk factor. Pan Afr Med J 2011; 10: 1-8.

41. Rowe A, Obeiro J, Newbold CI, Marsh K. Plasmodium falciparum rosetting is associated with malaria severity in Kenya. Infect Immun 1995. 63: 2323-2326.

42. Carlson J, Helmby H, Hill AV, Brewster D, Greenwood BM, Wahlgren M. Human cerebral malaria: association with erythrocyte rosetting and lack of anti-rosetting antibodies. Lancet $1990^{\circ}$ 336: $1457-1460$.

43. Vigan-Womas I, Guillotte M JA, Hessel A, Raynal B, England P, Cohen JH, et al. Structural basis for the ABO blood-group dependence of Plasmodium falciparum rosetting. PLoS Pathog 2012; 8: e1002781.

44. Chen Q, Barragan A, Fernandez V, Sundström A, Schlichtherle M, Sahlén A, et al. Identification of Plasmodium falciparum Erythrocyte Membrane Protein 1 (PfEMP1) as the rosetting ligand of the malaria parasite P. falciparum. J Exp Med 1998; 187: 15-23.

45. Oberli A, Slater LM, Cutts E, Brand F, Mundwiler-Pachlatko E, Rusch S, et al. A Plasmodium falciparum PHIST protein binds the virulence factor PfEMP1 and comigrates to knobs on the host cell surface. FASEB J 2014; 28: 4420-4433.

46. Lee WC, Malleret B, Lau YL, Mauduit M, Fong MY, Cho JS, et al. Glycophorin C (CD236R) mediates vivax malaria parasite resetting to normocytes. Blood 2014; 123: e100-e109.

47. Chitnis CE, Miller LH. Identification of the erythrocyte binding domains of Plasmodium vivax and Plasmodium knowlessi proteins involved in erythrocyte invasion. J Exp Med 1994; 180: 497-506.

48. Gupte SC, Patel AG, Patel TG. Association of $A B O$ groups in malaria infection of variable severity. J Vector Borne Dis 2012; 49: 78-81.

49. Bagudai S, Majhi B, Reddy KS. ABO BLOOD Group System as a Prognostic Indicator in Falciparum Malaria of Paediatric age Group. SEAJCRR 2015; 4: 1617-1622.

50. Rowe JA. Revealing the secrets of malaria parasite interaction with blood group A sugars. Pathog Glob Health 2013; 107: 45. 